\title{
Improving Mobility on Segments with Closely Spaced Intersections in Urban Areas
}

\author{
Stephen A. Arhin*, Adam Gatiba, Melissa Anderson, Melkamsew Ribbisso, Babin Manandhar \\ Howard University Transportation Research Center, Washington DC, USA \\ Email: *saarhin@howard.edu
}

How to cite this paper: Arhin, S.A., Gatiba, A., Anderson, M., Ribbisso, M. and Manandhar, B. (2019) Improving Mobility on Segments with Closely Spaced Intersections in Urban Areas. Open Journal of Civil Engineering, 9, 46-56.

https://doi.org/10.4236/ojce.2019.91004

Received: February 16, 2019

Accepted: March 16, 2019

Published: March 19, 2019

Copyright (c) 2019 by author(s) and Scientific Research Publishing Inc. This work is licensed under the Creative Commons Attribution International License (CC BY 4.0).

http://creativecommons.org/licenses/by/4.0/

\begin{abstract}
In this research, a strategy to improve mobility and reduce delay on road segments is explored via modeling and simulation. Thirty selected corridors with combination of signalized and unsignalized intersections were identified for this study. Each segment consists of at least one AWSC and two signalized intersections at which field data were obtained (lane configurations, signal timing, traffic volumes, etc.). The selected AWSC intersections on the segments were within $305 \mathrm{~m}$ (1000 feet) from the upstream or downstream signalized intersections. Synchro software program was utilized to model the existing condition of the segments based on which the strategy for mobility improvement was explored. The field data were used as input in Synchro software application to model two scenarios: existing or the "before" scenario, and the "after" scenario. The unsignalized intersections were signalized (and optimized) in the "after" scenario. The measures of effectiveness used to assess the efficiency of the strategy were average travel speed, control delay and $95^{\text {th }}$ percentile queue length. The analyses were conducted for both the morning (AM) and evening (PM) peak periods. The results of the analyses showed reductions in control delay and $95^{\text {th }}$ percentile queue lengths that were statistically significant, while the average travel speed of vehicles significantly increased at $5 \%$ level of significance. The evaluation determined that the signalization of some unsignalized intersections (which are $305 \mathrm{~m}$ or less from existing signalized intersections) may improve mobility despite the fact that these locations do not meet the MUTCD warrants for signalization. These findings would aid transportation engineers and planners to consider and evaluate this option when making decisions on signalization of intersections in urban areas.
\end{abstract}

\section{Keywords}

Mobility, Throughput, Control Delay, Average System, Travel Speed, Level of Service 


\section{Introduction}

Mobility continues to be of grave concern due to the rapid population growth in urban centers. Between 2015 and 2020, the urban population around the world is expected to grow approximately $1.84 \%$ per year. As the population in urban areas continues to increase, the capacities of road networks are being exceeded, resulting in the increase in travel time and delay of most commuters. Federal and local government agencies are continually exploring strategies to reduce congestion and improve mobility on road corridors. Despite the implementation of many measures, congestion continues to persist causing billions of dollars in lost revenue and pollution of the environment. In Washington DC, commuters spend on average, 60 hours in traffic annually. This is equivalent to about 3 billion dollars to the region's economy. Similarly, commuters in Los Angeles, California, experienced on average 104 hours of traffic delay annually. It is increasingly becoming difficult to expand existing physical infrastructure to improve mobility. In view of that more cost-effective measures which involve altering or improving traffic operational characteristics of road networks to increase capacity are encouraged [1].

In urban areas, congestion mostly originates at or near intersections. The road networks of such jurisdictions consist of a combination of signalized and unsignalized intersections which have a tendency of affecting throughput and mobility. The warrants prescribed in the Manual on Traffic Control Devices (MUTCD) need to be met for an intersection to be signalized. The effect on mobility may be compounded by All-Way Stop Control (AWSC) intersections which do not meet the MUTCD warrants and are in close proximity to signalized intersections. In this research a strategy to improve the mobility of vehicles and reduce delay is explored via modeling and simulation. Thirty selected corridors with combinations of signalized and unsignalized intersections were identified for this study where each segment consists of at least one AWSC and two signalized intersections at which field data collection was conducted (lane configurations, signal timing, traffic volumes, etc.). The selected AWSC intersections for this study were within $305 \mathrm{~m}$ from the upstream or downstream signalized intersections. Synchro software program was utilized to model the existing condition of the segments based on which the strategy for mobility improvement was explored.

The time and cost to travel from one place to the other is referred to as mobility. The Urban Mobility Report reports that congestion cost the United States about $\$ 87$ billion annually and the cost per person increased from \$290 in 1982 to $\$ 750$ in 2010. Also, billions of gallons of fuel have been wasted due to congestion. The cost of congestion is estimated to increase to $\$ 186$ billion by 2030 [2]. Joe Cortright et al. (2010), conducted a study to explore the key role that land use and variations in travel distances play in determining how long Americans spend in peak hour travels. The authors concluded that in the best performing cities, a typical traveler spends 40 hours less per year during peak hour travel 
than the average American. Such cities include Chicago, Portland and Sacramento. Residents of the worse performing cities spend as much as 240 hours per year [3].

Washington DC is a highly urbanized city and is ranked the 6th most congested city in the United States and it ranks $18^{\text {th }}$ across the world. Residents of Washington DC spend, on average, 61 hours in traffic annually [4]. The causes of traffic congestion and for that matter, poor mobility have been widely attributed to high vehicular volumes and inadequate road networks. However, in urban centers the frequent interruption of traffic flow contributes immensely to congestion. These interruptions mostly originate at or near intersections. Congestion is aggravated when intersection control devices on road segments are uncoordinated. It is generally recommended that intersections in urban areas are spaced a minimum of 805 meters ( 0.5 miles) [5].

When intersections are controlled by either YIELD or STOP signs they said to be unsignalized, whereas they are signalized when controlled by traffic signals. In urban areas, most of the roadway networks consist of combinations of unsignalized and signalized intersections which may impact mobility. Unsignalized intersections usually form most of the intersections on road networks. In Washington DC for instance, of the 7700 intersections, only 1450 are signalized while the remaining are unsignalized. The warrants for signalizing an intersection are prescribed in the Manual on Uniform Traffic Control Devices (MUTCD). Section 1A-09 of the MUTCD asserts that the decision to use a particular traffic control device at a specified location should be based on the application of engineering judgment and study, both of which shall be performed by or under the supervision an engineer [6]. The criteria in these warrants are hardly met by most intersections. On corridors where unsignalized intersections are in close proximity to signalized intersections, the effect on mobility can be adverse.

\subsection{Improving Mobility on Road Corridors}

Both short-term and long-term strategies have been proposed and/or implemented to reduce congestion in urban areas. These strategies include adding new facilities or making operational changes to improve system performance. The addition of new facilities generally increases the capacity of the road network. However, this measure is cost intensive and in the long term will generate more traffic. The less cost intensive measures involve improving the existing infrastructure to improve capacity. Kumarage (2004) reports that one effective measure in improving urban mobility is the signalization of unsignalized intersections, street widening of bottlenecks or providing grade separated intersections [7]. However, the study did not indicate the conditions under which signalization of unsignalized intersections were warranted.

In addition, measures such as redesigning existing infrastructure to improve mobility have also been explored. These include the conversion of travel lanes to bus lanes and high occupancy, providing pedestrian only streets and removal of 
on-street parking. Also, operational changes have been implemented to improve mobility. The introduction of reversible lanes during peak periods has been determined to increase the capacity of roadways. Available lane capacity is obtained from the off-peak direction and added to the peak directional flow for congestion reduction purposes. Reversible lanes are effective in handling morning and evening peak commute periods, special event traffic, as well as during construction and maintenance activities. Variable message signs are often used to identify the direction of the reversed lanes at selected times [8].

When the existing signal timing plans at some intersections no longer efficiently facilitate the movement vehicles on road corridors, signal re-timing is sometimes employed to ease congestion. Signal re-timing has the capability of optimizing traffic signal operation. In Washington DC, a study showed a 13\% reduction in delay is experienced on segments where traffic retiming was implemented. Thus, fewer stops, and lower fuel consumption is achieved. However, this measure is most effective on corridors with normalized traffic patterns [9]. This study did not consider the effects of the proximity of alternating signalized and unsignalized intersections on a road segment.

Further, the Federal Highway Administration (FHWA) has recommended that for unsignalized intersections in close proximity to signalized intersections, it is appropriate to re-time adjacent signals to create adequate gaps in traffic for easier turning maneuvers at unsignalized intersections. Moreover, automated real-time systems and innovative signs to inform drivers of suitable gaps for turning or crossing maneuvers are also recommended [10].

In recent times more, advanced techniques involving the use of Intelligent Transportation Systems (ITS) have been deployed to improve mobility and reduce congestion in urban areas. These systems provide communication links that give real-time travel conditions and emergency management information to the general public, emergency response providers, and transportation agencies. Also, regional traffic management technologies, such as a closed-circuit television, are used to significantly improve mobility by revising traffic signal timings at intersections.

\subsection{Mobility Performance Indicators}

Mobility can be measured in various ways. Traffic engineers utilize traffic data and perform operational analyses to obtain various performance indicators which can be used to measure congestion and consequently mobility on road segments. These performance indicators include level of service, queue lengths, system travel speeds, and average vehicular delays.

\section{Methodology}

\subsection{Segment Selection}

Thirty (30) segments on arterial and collector streets in Washington, DC were selected for this study. Each segment consists of at least one AWSC intersection 
and two signalized intersections. The segments were selected such that no signalized intersections were in succession and have at most two AWSC intersections in between. Figure 1 shows a typical configuration of a segment.

\subsection{Data Collection}

Field data collection was conducted at the thirty (30) selected segments on typical weekdays (Tuesday, Wednesday and Thursday) in 2017. Data collection was deferred at any of the intersections or segments where road maintenance or construction was ongoing until it was completed. The following traffic operations data were obtained:

1) Vehicular and Pedestrian Volumes

Video cameras were installed at the same time at each intersection within each segment of study. The video recordings were done from 6:30 AM to 6:30 PM on the said weekdays. The pedestrian and vehicular volumes were extracted via video playback, using turning movement count boxes. The AM and PM peak hour volumes, peak hour factors and heavy vehicle percentages were computed using PetraPro software.

2) Signal Timing Data

Existing signal timing information for the signalized intersections was obtained from the District Department of Transportation (DDOT). In the absence of signal timing data, filed measurements were conducted using a stop watch. The green, change and clearance timing intervals for each phase were measured three (3) times after which the averages were then computed. The cycle lengths were then calculated by summing the average green and yellow intervals times.

3) Geometric Characteristics

The following geometric characteristics were documented during field assessment: segment lengths, lane widths, number of lanes, presence of restrictions of left turns, presence of parking on the street, land use and grades of intersection, presence of right turns on red restrictions.

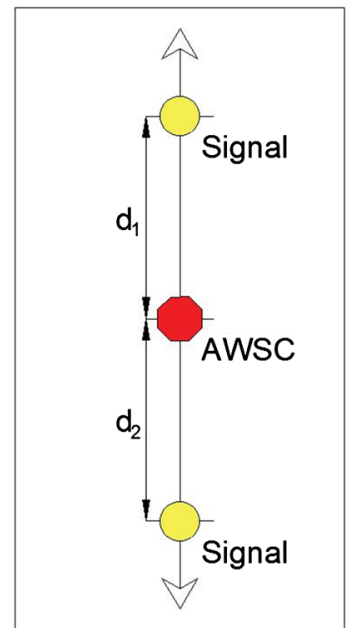

Figure 1. Typical study configuration. 


\subsection{Data Analysis}

\subsubsection{Level of Service (LOS)}

Data obtained were used as input in Synchro 9 to simulate two scenarios: the "before" scenario which is the existing conditions and the "after" scenario, where the AWSC intersections were signalized and coordinated with the existing signalized intersections. Synchro's optimization feature was used in the "after": scenario which also provides signal coordination. The resulting intersections' LOS, as well as the segments' measures of effectiveness (MOEs) for both scenarios were extracted for comparison. The performance of the segments in the "before" and "after" scenarios were assessed using the following MOEs: control delay, average system travel speed and the $95^{\text {th }}$ percentile queue length. These are defined as follows:

Control Delay: Control delay is the total of uniform delay, incremental delay and initial delay with respect to a progression factor. Control delay includes movements at slower speeds and stops on intersection approaches as vehicles move up in queue position or slow down upstream of an intersection.

Average System Travel Speed: This is the average speed of vehicles on the segments. This speed takes into consideration the intersections spacing, the running time between intersections, and the control delay of vehicles at each intersection. Thus, the simulated travel speeds do not usually correspond to speed measurements made from end-to-end travel time runs that measure a small subset of the possible origin-destination combinations along a segment.

$95^{\text {th }}$ Percentile Queue Length: This is the queue length that has only a 5 -percent probability of being exceeded during the analysis time period.

\subsubsection{Statistical Analysis}

Descriptive statistics of the MOEs extracted from the Synchro results were computed and used for further analysis. These statistics include the mean, median, and standard deviation.

\subsubsection{Test of Hypothesis}

The mean is the primary test statistic used for the comparison in this study. The hypothesis that the "after" scenario would result in an improvement of mobility along the segment over the "before" scenario was tested at 5\% level of significance. It is hypothesized that following improvements will occur in the "after" scenario:

Reduction in the mean control delay along the segments

Increase in the mean system travel speed along the segments and

Reduction in the $95^{\text {th }}$ percentile queue lengths at the existing signalized intersections

1) Reduction in Control Delays

The hypothesized reduction in control delay in the "after" scenario is mathematically expressed as

$$
\mathrm{H}_{0}: \bar{X}_{2} \geq \bar{X}_{1}
$$




$$
\mathrm{H}_{1}: \bar{X}_{2}<\bar{X}_{1}
$$

where

$\bar{X}_{1}=$ mean control delay in the "before" scenario.

$\bar{X}_{2}=$ mean control delay in the "after" scenario.

2) Increased Average System Travel Speeds

The hypothesized increment in average system travel speeds in the "after" scenario is mathematically expressed as

$$
\begin{array}{ll}
\mathrm{H}_{0}: & \bar{Y}_{2} \geq \bar{Y}_{1} \\
\mathrm{H}_{1}: & \bar{Y}_{2}<\bar{Y}_{1}
\end{array}
$$

where,

$\bar{Y}_{1}=$ mean travel speed in the "before" scenario.

$\bar{Y}_{2}=$ mean travel speed in the "after" scenario.

3) Reduced $95^{\text {th }}$ Percentile Queue Lengths

The hypothesized reduction in the $95^{\text {th }}$ percentile queue lengths at the existing signalized intersections in the "after" scenario is mathematically expressed as

$$
\begin{aligned}
& \mathrm{H}_{0}: \quad \bar{Z}_{2} \geq \bar{Z}_{1} \\
& \mathrm{H}_{1}: \quad \bar{Z}_{2}<\bar{Z}_{1}
\end{aligned}
$$

where,

$\bar{Z}_{1}=$ mean $95^{\text {th }}$ percentile queue length in the "before" scenario.

$\bar{Z}_{2}=$ mean $95^{\text {th }}$ percentile queue length in the "after" scenario.

\section{Results}

\subsection{LOS Analysis Results}

Level of Service (LOS) analysis was conducted based on procedures in the 2016 Highway Capacity Manual (HCM), using Synchro 9. The LOS of each AWSC intersection was analyzed for both the "before" and "after" scenarios. However, the LOS of the signalized intersections were assessed for only the "before" scenario, since the conditions at those intersections were kept the same in the "after" scenario. Also, the segments' MOEs (control delay, travel speed and $95^{\text {th }}$ percentile queue length) were obtained from the Synchro results. The LOS for the "after" scenario of the AWSC intersections showed improvements over the "before" scenario as can be observed in the results. The system travel speeds of the segments in the "after" scenario either remained the same or increased compared to the speeds in the "before" scenario.

\subsection{Statistical Analysis}

Tables 1-3 present summaries of the descriptive statistical analyses for the segment MOEs (control delay, average travel speed and $95^{\text {th }}$ percentile queue length). The reported descriptive statistics are the mean, median, standard deviation and $95 \%$ confidence interval. The mean control delays for the thirty (30) segments were higher for the "before" scenario compared to the "after" scenario 
as shown in Table 1 . The mean control delay was $29.93 \mathrm{sec} / \mathrm{v}$ for the AM peak period. However, this reduced to $27.23 \mathrm{sec} / \mathrm{v}$ for the "after" scenario. The mean control delay in the PM peak period was $28.80 \mathrm{sec} / \mathrm{v}$. Likewise, this was reduced to $23.80 \mathrm{sec} / \mathrm{v}$ for the "after" scenario. The highest control delay of $133 \mathrm{sec} / \mathrm{v}$ was recorded during the AM peak, while the lowest control delay of $10 \mathrm{sec} / \mathrm{v}$ was recorded during the PM peak period for the "after" scenario.

Table 2 shows the summary of the descriptive statistics of the average travel speeds on the segments. The average travel speeds were lower for the "before" scenario than the "after" scenario. During the AM peak period, the mean travel speed was $10.46 \mathrm{~km} / \mathrm{hr}$, while in the "after" scenario the mean travel speed increased to $11.47 \mathrm{~km} / \mathrm{hr}$. During the PM peak period the mean travel speed was $10.51 \mathrm{~km} / \mathrm{hr}$. Similarly, an increase in the travel speeds was recorded in the "after" scenario. The mean travel speed increased to $11.15 \mathrm{~km} / \mathrm{hr}$. The highest average segment travel speed of $20.92 \mathrm{~km} / \mathrm{hr}$ occurred during the PM peak, while the lowest average segment speed of $1.61 \mathrm{~km} / \mathrm{hr}$ occurred during the AM peak period. The summary of the descriptive statistics of the $95^{\text {th }}$ percentile queue length at the existing signalized intersection on the segments are shown in Table 3. Higher $95^{\text {th }}$ queue lengths were recorded in the "before" scenario than the "after" scenario. The mean queue length was $36.67 \mathrm{~m}$ (120.33 feet) for the AM peak period. This decreased to $32.49 \mathrm{~m}$ (106.60 feet) for the "after" scenario. Forty-six percent of the intersections recorded reduced queue lengths in the "after" scenario, while $44 \%$ did not vary. Only $10 \%$ of the intersections recorded higher queue lengths in the "after" scenario. During the PM peak period the mean queue length

Table 1. Summary of descriptive statistics for control delay.

\begin{tabular}{|c|c|c|c|c|c|c|c|c|}
\hline \multirow{3}{*}{ Peak Period } & \multirow{3}{*}{ MOE } & \multicolumn{7}{|c|}{ Descriptive Statistics } \\
\hline & & \multirow{2}{*}{ Mean } & \multirow{2}{*}{ Stand. Dev. } & \multirow{2}{*}{ Median } & \multirow{2}{*}{ Min. Value } & \multirow{2}{*}{ Max. Value } & \multicolumn{2}{|c|}{ 95\% Confidence Interval } \\
\hline & & & & & & & Lower Bound & Upper Bound \\
\hline \multirow{2}{*}{$\mathrm{AM}$} & "Before" Control Delay (sec/v) & 29.93 & 23.72 & 23.00 & 11 & 133 & 21.08 & 38.79 \\
\hline & “After” Control Delay (sec/v) & 27.23 & 23.77 & 18.00 & 11 & 130 & 18.36 & 36.11 \\
\hline \multirow{2}{*}{ PM } & "Before" Delay (sec/v) & 28.07 & 18.05 & 23.00 & 11 & 98 & 21.33 & 34.81 \\
\hline & “After” Control Delay (sec/v) & 23.80 & 16.39 & 19.50 & 10 & 91 & 17.68 & 29.92 \\
\hline
\end{tabular}

Table 2. Summary of descriptive statistics for average segment travel speed.

\begin{tabular}{|c|c|c|c|c|c|c|c|c|}
\hline \multirow{3}{*}{ Peak Period } & \multirow{3}{*}{ MOE } & \multicolumn{7}{|c|}{ Statistic } \\
\hline & & \multirow{2}{*}{ Mean } & \multirow{2}{*}{ Stand. Dev. } & \multirow{2}{*}{ Med } & \multirow{2}{*}{ Min. Value } & \multirow{2}{*}{ Max. Value } & \multicolumn{2}{|c|}{ 95\% Confidence Interval } \\
\hline & & & & & & & Lower Bound & Upper Bound \\
\hline \multirow{2}{*}{$\mathrm{AM}$} & "Before" Average Travel Speed $(\mathrm{km} / \mathrm{hr})$ & 10.46 & 4.09 & 10.46 & 1.61 & 19.31 & 8.93 & 11.99 \\
\hline & “After” Average Travel Speed (km/hr) & 11.47 & 4.65 & 11.27 & 1.61 & 20.92 & 9.75 & 13.21 \\
\hline \multirow{2}{*}{$\mathrm{PM}$} & "Before" Average Travel Speed $(\mathrm{km} / \mathrm{hr})$ & 10.46 & 4.15 & 11.26 & 4.83 & 20.92 & 8.92 & 12.01 \\
\hline & “After" Average Travel Speed (km/hr) & 11.15 & 3.94 & 11.26 & 4.83 & 20.92 & 9.69 & 12.63 \\
\hline
\end{tabular}


Table 3. $t$-Test results- $95^{\text {th }}$ percentile queue lengths.

\begin{tabular}{|c|c|c|c|c|c|c|c|c|}
\hline \multirow{3}{*}{ Peak Period } & \multirow{3}{*}{ MOE } & \multicolumn{7}{|c|}{ Descriptive Statistics } \\
\hline & & \multirow{2}{*}{ Mean } & \multirow{2}{*}{ Std Dev. } & \multirow{2}{*}{ Med. } & \multirow{2}{*}{ Min. Value } & \multirow{2}{*}{ Max. Value } & \multicolumn{2}{|c|}{ 95\% Confidence Interval } \\
\hline & & & & & & & Lower Bound & Upper Bound \\
\hline \multirow{2}{*}{$\mathrm{AM}$} & "Before" $95^{\text {th }}$ Per. Queue Length $(\mathrm{m})$ & 36.68 & 30.8 & 23.47 & 0.3 & 140.82 & 28.1 & 45.25 \\
\hline & “After" $95^{\text {th }}$ Per. Queue Length $(\mathrm{m})$ & 32.49 & 30.28 & 20.72 & 0.3 & 135.33 & 24.06 & 40.92 \\
\hline \multirow{2}{*}{$\mathrm{PM}$} & "Before" $95^{\text {th }}$ Per. Queue Length (m) & 42.05 & 32.56 & 30.02 & 0.3 & 166.12 & 25.8 & 44.06 \\
\hline & "After" $95^{\text {th }}$ Per. Queue Length $(\mathrm{m})$ & 34.93 & 32.78 & 38.56 & 0.91 & 166.12 & 32.96 & 51.11 \\
\hline
\end{tabular}

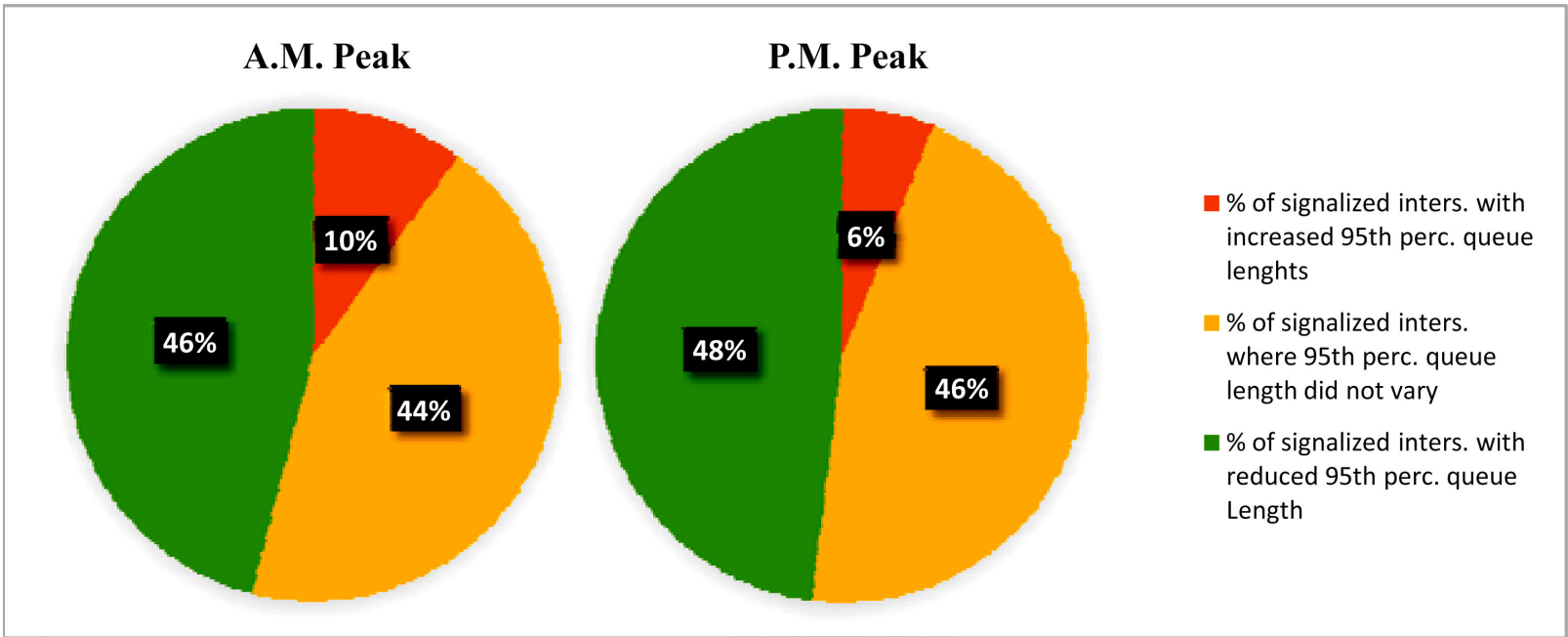

Figure 2. Changes in $95^{\text {th }}$ percentile queue lengths of the intersections.

was $42.05 \mathrm{~m}$ (137.96 feet). Similarly, there was a decrease in queue length to $34.93 \mathrm{~m}$ ( $114.60 \mathrm{feet})$ for the "after" scenario. While $48 \%$ of the intersections recorded reduced queue lengths in the "after scenario", $46 \%$ did not vary. The remaining $6 \%$ of the intersections recorded higher queue lengths in the "after scenario". Figure 2 shows the proportion of changes in the $95^{\text {th }}$ percentile queue in the "after" scenario. It can be observed from the figure that during both peak periods, a higher proportion of the signalized intersections had a reduction in the $95^{\text {th }}$ percentile queue length for the "after" scenario.

\subsection{Results of Test for Hypothesis}

The statistically significant differences in the mean "before" and "after" segments' MOEs were tested for using the t-test. The results are presented in the following sections.

\subsection{1. t-Test to Test for Statistically Significant Differences in Control Delay}

The results of the t-test to test for statistically significant differences in the control delays of the "before" and "after" scenarios are presented in Table 4. It can be seen from the table that there was a mean difference of $2.70 \mathrm{sec} / \mathrm{v}$ in control 
delays of the "before" and "after" scenarios. This difference was determined to be statistically significant at $5 \%$ level of significance, $t_{29}=3.41$ and $p=0.002$. Also, there was statistically significant change in mean difference in control delays of the "before" and "after" scenarios, $\mathrm{t}_{29}=3.52$ and $\mathrm{p}=0.001$, for the PM peak period.

\subsection{2. t-Test to Test for Statistically Significant Differences in Average System Travel Speeds}

Table 5 presents the results of the t-test to test for statistically significant differences in the control delays of the "before" and "after" scenarios. It can be observed from the table that the difference in average system travel speed was $-10.19 \mathrm{~km} / \mathrm{hr}$ during the AM peak period. This difference was determined to be statistically significant, $t_{29}=-2.92$ and $p=0.007$. Also, the PM peak period results show that there was a statistically significant difference in average travel system speeds $(-0.697 \mathrm{~km} / \mathrm{hr}), \mathrm{t}_{29}=-2.21$ and $\mathrm{p}=0.035$.

\subsection{3. t-Test to Test for Statistically Significant Differences in the $95^{\text {th }}$ Percentile Queue Lengths}

Table 6 presents the results of the t-test to test for statistically significant differences in the $95^{\text {th }}$ percentile queue lengths of the "before" and "after" scenarios. It can be observed from the table that the mean difference in $95^{\text {th }}$ percentile queue lengths of the "before" and "after" scenarios was $4.18 \mathrm{~m}$ (13.73 feet) during the AM peak period. This difference was determined to be statistically non-significant at $95 \%$ confidence interval, $\mathrm{t}_{51}=1.74$ and $\mathrm{p}=0.088$. However, the results of the PM peak period show a statistically significant difference in mean difference in the $95^{\text {th }}$ percentile queue lengths of the "before" and "after" scenarios, $t_{51}=3.88$ and $\mathrm{p}<0.00$.

Table 4, $t$-Test results-control delay.

\begin{tabular}{|c|c|c|c|c|c|c|}
\hline Peak Period & Variable & Mean Diff. & Std. Dev & $\mathrm{t}$ & df & Sig. \\
\hline $\mathrm{AM}$ & "Before" Control Delay_“After" Control Delay & 2.70 & 4.34 & 3.41 & 29 & 0.002 \\
\hline PM & "Before” Control Delay_ “After" Control Delay & 4.27 & 6.65 & 3.52 & 29 & 0.001 \\
\hline
\end{tabular}

Table 5. $t$-Test results-average system travel speed

\begin{tabular}{|c|c|c|c|c|c|c|}
\hline Peak Period & Variable & Mean Diff. & Std. Dev & $\mathrm{t}$ & df & Sig. \\
\hline $\mathrm{AM}$ & "Before” Average Travel Speed_“After” Average Travel Speed (km/hr) & -10.19 & 1.92 & -2.92 & 29 & 0.007 \\
\hline PM & "Before” Average Travel Speed_“After” Average Travel Speed (km/hr) & -0.697 & 1.72 & -2.21 & 29 & 0.035 \\
\hline
\end{tabular}

Table 6. $t$-Test results $-95^{\text {th }}$ percentile queue lengths.

\begin{tabular}{|c|c|c|c|c|c|c|}
\hline Peak Period & Variable & Mean Diff. & Std. Dev & $\mathrm{t}$ & $\mathrm{df}$ & Sig. \\
\hline $\mathrm{AM}$ & "Before" $95^{\text {th }}$ Per. Queue Length (ft) - “After" 95 ${ }^{\text {th }}$ Per. Queue Length (ft) & 4.18 & 17.36 & 1.74 & 51 & 0.088 \\
\hline PM & "Before" $95^{\text {th }}$ Per. Queue Length (ft)-“After" 95 ${ }^{\text {th }}$ Per. Queue Length (ft) & 7.12 & 13.25 & 3.88 & 51 & 0.000 \\
\hline
\end{tabular}




\section{Conclusion and Recommendations}

The general significant improvement in the segment selected MOEs (control delay, average travel speed and $95^{\text {th }}$ percentile queue length) in the "after" scenario indicates an overall improvement in mobility. Moreover, the improvements were mostly determined to be statistically significant at $5 \%$ level of significance. The research revealed that, signalizing some unsignalized intersections (which are $305 \mathrm{~m}$ or less from existing signalized intersections) may improve mobility and throughput even though the intersections may not meet the MUTCD warrants for signalization. This study explored the effects of signalizing AWSC intersections to improve mobility along segments in urban areas via simulation. It is therefore recommended that a selected number of segments be used as test-beds for validation of the outcome of this study. Also, future studies could consider longer segments with combinations of AWSCs and signalized intersections.

\section{Conflicts of Interest}

The authors declare no conflicts of interest regarding the publication of this paper.

\section{References}

[1] Guerrini, F. (2014) Traffic Congestion Costs Americans \$124 Billion a Year. https://www.forbes.com/sites/federicoguerrini/2014/10/14/traffic-congestion-costsamericans-124-billion-a-year-report-says/\#20f1466cc107

[2] Schrank, D., Eisele, B., Lomax, T. and Bak, J. (2010) 2010 Urban Mobility Scorecard. The Texas A\&M Transportation Institute and INRIX.

[3] Cortright, J. (2010) Measuring Urban Transportation Performance: A Critique of Mobility Measures and a Synthesis. CEOs for Cities.

[4] Cookson, G. (2018) NRIX Global Traffic Scorecard, INRIX Research.

[5] Center for Transportation Research and Education. Iowa State University (2007) Access Management Toolkit, Intersection Spacing and Traffic Signal Spacing. http://www.ctre.iastate.edu/research/access/toolkit/4.pdf

[6] Manual on Uniform Traffic Control Devices 2010 Edition (2010).

[7] Kumarage (2004) Urban Traffic Congestion: The Problem \& Solutions. The Asian Economic Review.

[8] Management, Traffic, System Efficiency, and Reversible Traffic Lanes. Reversible Traffic Lanes (2014) Texas A\&M Transportation Institute.

[9] Srinivasa, S. (2004) The Benefits of Retiming Traffic Signals. Institute of Transportation Engineers: 26-29.

[10] Federal Highway Administration (2003) Unsignalized Intersection Safety Strategies. NCHRP Report 500/Volume 5: A Guide for Addressing Unsignalized Intersection Collisions. 